\title{
Transcription of Leishmania major U2 small nuclear RNA gene is directed by extragenic sequences located within a tRNA-like and a tRNA-Ala gene
}

\author{
Saúl Rojas-Sánchez ${ }^{1}$, Elisa Figueroa-Angulo ${ }^{1}$, Rodrigo Moreno-Campos ${ }^{1}$, Luis E. Florencio-Martínez ${ }^{1}$,
} Rebeca G. Manning-Cela ${ }^{2}$ and Santiago Martínez-Calvillo ${ }^{1^{*}}$

\begin{abstract}
Background: Leishmania and other trypanosomatid parasites possess atypical mechanisms of gene expression, including the maturation of mRNAs by trans-splicing and the involvement of RNA Polymerase III in transcription of all snRNA molecules. Since snRNAs are essential for trans-splicing, we are interested in the study of the sequences that direct their expression. Here we report the characterization of L. major U2 snRNA promoter region.

Results: All species of Leishmania possess a single U2 snRNA gene that contains a divergently-oriented tRNA-Ala gene in the upstream region. Between these two genes we found a tRNA-like sequence that possesses conserved boxes A and B. Primer extension and RT-qPCR analyses with RNA from transiently-transfected cells showed that transcription of L. major U2 snRNA is almost abolished when boxes A and B from the tRNA-like are deleted or mutated. The levels of the U2 snRNA were also highly affected when base substitutions were introduced into box $B$ from the tRNA-Ala gene and the first nucleotides of the U2 snRNA gene itself. We also demonstrate that the tRNA-like is transcribed, generating a main transcript of around 109 bases. As pseudouridines in snRNAs are required for splicing in other organisms, we searched for this modified nucleotide in the L. major U2 snRNA. Our results show the presence of six pseudouridines in the U2 snRNA, including one in the Sm site that has not been reported in other organisms.

Conclusions: Four different regions control the transcription of the U2 snRNA gene in L. major. boxes $A$ and $B$ from the neighbor tRNA-like, box B from the upstream tRNA-Ala gene and the first nucleotides of the U2 snRNA. Thus, the promoter region of $L$. major $\mathrm{U} 2$ snRNA is different from any other promoter reported for snRNAs. Pseudouridines could play important roles in L. major U2 snRNA, since they were found in functionally important regions, including the branch point recognition region and the Sm binding site.
\end{abstract}

Keywords: Leishmania, U2 snRNA, Promoter region, tRNA-like, Pseudouridine

\section{Background}

The information in eukaryotic protein-coding genes is frequently interrupted by introns, which have to be removed from the precursors of the mRNAs before they can be translated. The process in which introns are removed and exons are joined together is called splicing. This procedure is carried out in a stepwise coordinated fashion by a large

\footnotetext{
* Correspondence: scalv@campus.iztacala.unam.mx

${ }^{1}$ Unidad de Biomedicina, Facultad de Estudios Superiores Iztacala,

Universidad Nacional Autónoma de México, Av. de los Barrios 1, Col. Los

Reyes Iztacala, Tlalnepantla, Edo. de México CP 54090, Mexico

Full list of author information is available at the end of the article
}

ribonucleoprotein complex, named spliceosome, that consists of around 200 proteins [1] and five major small nuclear RNAs (snRNAs): U1, U2, U4, U5 and U6 [2, 3]. Each snRNA binds several specific proteins, as well as common proteins, to form a small nuclear ribonucleoprotein particle (snRNP). Among these proteins, the $\mathrm{Sm}$ proteins form a donut-shaped heptamer that interacts with a single-stranded region, the $\mathrm{Sm}$ site, within the U1, U2, U4 and U5 snRNAs [4]. On the other hand, the U6 snRNA binds $\mathrm{Sm}$-like proteins that recognize a U-tract sequence located at the 3' end of the RNA [5]. 
The sequence and secondary structure of snRNAs are highly conserved, even between phylogenetically distant organisms [6]. All snRNAs are extensively posttranscriptionally modified and pseudouridine is the most abundant modified nucleotide in these RNA molecules [7]. Notably, most pseudouridines are located in functionally important regions of the snRNAs. In fact, several studies have demonstrated that pseudouridines play important roles in both snRNP assembly and splicing $[8,9]$.

Two different RNA polymerases (RNAP) are involved in the synthesis of snRNAs: U1 to U5 are transcribed by RNAP II, whereas U6 is transcribed by RNAP III [10]. In humans, the snRNA core promoter recognized by both RNAP II and III is composed of two elements: a proximal sequence element that is located between nucleotides -50 to -65 , and a distal sequence element lying between positions -200 to -220 , in relation to the transcription start site (TSS). Additionally, the RNAP III promoter contains a TATA box element situated around position $-25[11,12]$.

Although splicing is widely conserved among eukaryotes, some organisms possess special forms of this mechanism to process mRNAs. For instance, proteincoding genes in Leishmania and other protozoan parasites that belong to the family Trypanosomatidae are organized into polycistronic gene clusters that are transcribed by RNAP II to generate polycistronic transcripts; and mature mRNAs are produced from these precursors by trans-splicing and polyadenylation. Trans-splicing is a process that adjoins a capped 39-nucleotide miniexon or spliced leader to the $5^{\prime}$ termini of all the mRNAs [13]. Like cis-splicing, trans-splicing occurs via two transesterification reactions and requires the participation of snRNAs, but it involves the formation of a Y structure instead of a lariat intermediate [14, 15]. Although every snRNA has an essential participation in splicing, it has been shown that U2 and U6 snRNAs are key role players in the process, as they base-pair with each other to form the splicing catalytic core [16].

Another peculiarity of gene expression in trypanosomatids is that all snRNAs are transcribed by RNAP III [17]. Moreover, snRNA genes in Trypanosoma brucei have a divergently oriented tRNA gene, or a tRNA-like sequence, in their 5'-flanking region, and boxes $\mathrm{A}$ and $\mathrm{B}$ from the neighboring tRNA gene are essential for expression of the snRNAs [17-19]. Notably, the distance between box A from the tRNA gene and the snRNA gene is conserved, as it is usually $\sim 104 \mathrm{bp}$ in length [20, 21]. A third element located within the first 21 nucleotides of the snRNA coding region is also required for proper transcription initiation [17, 22]. However, not every snRNA gene needs all three sequence elements for transcription, as box $\mathrm{B}$ is dispensable for the in vitro synthesis of the U6 snRNA [22]. Also, in vivo expression of the U1 snRNA does not require an element located within the snRNA itself [19]. Moreover, box A of the associated tRNA gene is dispensable for U4 snRNA transcription in the related trypanosomatid Leptomonas collosoma [23].

Little attention has been paid to the study of snRNAs in Leishmania. In the present study we have analyzed the sequences that direct transcription of the U2 snRNA gene in L. major, the causative agent of cutaneous leishmaniasis in the Old World. As reported in other trypanosomatids, analysis of the genomic context of the U2 snRNA gene allowed us to identify a tRNA-like sequence located in the $5^{\prime}$ flanking region that contains typical boxes $\mathrm{A}$ and B. By performing RT-qPCR analysis we found that these two elements, together with an intragenic sequence, are required for transcription of the $\mathrm{U} 2$ snRNA. Unlike other snRNAs in trypanosomatids, our results showed that an additional box $\mathrm{B}$ that resides on a tRNA-Ala gene located 211 bp upstream the U2 snRNA gene, is also important for the optimal transcription of this gene in L. major.

\section{Methods}

Leishmania major culture and transfection

Promastigotes from L. major MHOM/IL/81/Friedlin (LSB-132.1) were grown in BM medium (1× M199 medium $\mathrm{pH}$ 7.2, containing $10 \%$ heat-inactivated fetal bovine serum, $0.25 \times$ brain heart infusion, $40 \mathrm{mM}$ HEPES, $0.01 \mathrm{mg} / \mathrm{ml}$ hemin, $0.0002 \%$ biotin, $100 \mathrm{IU} / \mathrm{ml}$ penicillin, $100 \mu \mathrm{g} / \mathrm{ml}$ streptomycin and $1 \times \mathrm{L}$-glutamine) at $26{ }^{\circ} \mathrm{C}$ and harvested in the mid-log phase. Electroporations were performed following the high-voltage protocol previously described [24]. Usually, 25-50 $\mu \mathrm{g}$ of test plasmid and cotransfection plasmid pLMRIB [25] were aliquoted into 4-mm gap cuvettes, and $500 \mu \mathrm{l}$ of cells $\left(2 \times 10^{8}\right.$ cells $\left./ \mathrm{ml}\right)$ were added to the cuvette and mixed. The cells were electroporated twice at $1500 \mathrm{~V}$ and $25 \mu \mathrm{F}$ (ECM 630 Electroporation System, BTX, Holliston, USA), pausing $10 \mathrm{~s}$ between pulses. Following electroporation, cells were transferred to $10 \mathrm{ml}$ of $\mathrm{BM}$ medium and incubated at $26{ }^{\circ} \mathrm{C}$, and total RNA was isolated $24 \mathrm{~h}$ post-transfection using TRI reagent (Sigma, St. Louis, USA).

\section{Bioinformatic analyses}

Sequence information from different trypanosomatids was obtained from the NCBI (http://www.ncbi.nlm.nih.gov/) and TriTrypDB (Release 27) (http://tritrypdb.org/ tritrypdb/) databases. BLAST searches were performed to obtain information for synteny maps and to search for U2-associated tRNA-like sequences. Alignments were generated using the MUSCLE (http://www.ebi.ac.uk/ Tools/msa/muscle/) [26] and DNAMAN (version 6, 
Lynnon Corporation, Quebec, Canada) programs and corrected manually. Secondary structure analysis was performed using the RNAfold Web Server (http://rna.tbi.univie.ac.at/cgi-bin/RNAfold.cgi) [27] with default parameters. Graphical representations of patterns within multiple sequence alignments were generated with the WebLogo application (http://weblogo.threeplusone.com/).

\section{Primer extension analysis}

Total RNA was analyzed by primer extension with $5^{\prime}$ end-labelled synthetic DNA oligonucleotides as described previously [28]. The following oligonucleotides were used: 3'endLmjU2 (5'-GAA AAA AGG AGT TGC TCC CCT GGA AAC GTG-3'), complementary to U2 nt 122 to 151; LmjU2tag-Rev (5'-ATT AGA GTC GAG GTC AGA CC-3'), complementary to a 15-nt tag sequence inserted between $\mathrm{U} 2$ nt 83 and 84 (tag sequence is underlined); and 5'LmjU2DcajaB (5'-ATC CGG CCC TGG TCT CCA AA-3'), complementary to tRNA-like nt 58 to 77 . In a typical experiment, 20 to $50 \mu \mathrm{g}$ of total RNA were hybridized to $1 \times 10^{5} \mathrm{cpm}$ of $\gamma$ - ${ }^{32} \mathrm{P}$-labelled oligonucleotide at $55{ }^{\circ} \mathrm{C}$ for $90 \mathrm{~min}$. The reaction volume was then increased to $50 \mu \mathrm{l}$ by the addition of a solution containing $39.5 \mu \mathrm{l}$ of RT buffer and $0.5 \mu \mathrm{l}$ of SuperScript III Reverse Transcriptase (Invitrogen, Carlsbad, USA). The reaction was incubated at $55{ }^{\circ} \mathrm{C}$ for $60 \mathrm{~min}$. Nucleic acids were precipitated and an aliquot of the products was separated on $8 \%$ polyacrylamide$8 \mathrm{M}$ urea gels and exposed to phosphor imaging plates. Autoradiograms were scanned on a Fujifilm FLA-5000 system (Fuji Photo Film Co., Ltd., Tokyo, Japan). When the U2 promoter region was analyzed, bands corresponding to a non-specific product were used to normalize samples of the U2 tagged-specific primer extension. Sequence ladders of U2, U2-tagged and tRNA-like sequences were obtained using a Sequenase 7-deaza-dGTP Sequencing Kit (USB, Cleveland, USA) as specified by the manufacturer. Constructs ptRNAU2 and pComp were used as templates in the sequencing reactions.

\section{5'-RACE analysis}

5' Rapid Amplification of cDNA Ends (5'-RACE) experiments were performed with $5 \mu \mathrm{g}$ of total RNA from $L$. major with a kit from Thermo Scientific (Waltham, USA). First strand cDNA was synthesized with primer 3'U2GST1 (5'-AAC GTG GAA CTC CAA GGA AA-3'), and PCR amplifications were performed with nested primer 3'U2GST2 (5'-CCT TGA GTT GTA ATT TCT AT3') and nested Abridged Anchor Primer, AAP (5'-GGC CAC GCG TCG ACT AGT ACG GGI IGG GII GGG IIG-3'). The nested PCR products were cloned into the pGEM-T Easy vector (Promega, Fitchburg, USA) and sequenced.

\section{RT-PCR analysis}

U2 snRNA transcription termination sites were mapped by poly(A) tailing of total RNA, as previously reported [29]. The cDNA was prepared with oligonucleotide Nested(dT) (5'-CCT CTG AAG GTT CAC GGA TCC ACA TCT AGA TTT TTT TTT TTT TTT TTT VN-3'). The first PCR was performed with primers U2Sa-5' (5'-ATA TCT TCT CGG CTA TTT AGC-3') and B1 (5'-CCT CTG AAG GTT CAC GGA T-3'), and the second PCR was done with primers U2qFw2 (5'-CTA TTT AGC TAA GAT CAT GTT TAT A-3') and B2 (5'-CAC GGA TCC ACA TCT AGA T-3'). The final PCR products were cloned into the pGEM-T Easy vector (Promega) and sequenced.

\section{Mapping of pseudouridines}

A primer extension-based pseudouridylation assay was carried out as previously described [30, 31]. Briefly, RNA from $5 \times 10^{8}$ cells was treated with $N$-cyclohexyl- $N^{\top}-\beta$ (4-methylmorpholinium)ethyl-carbodiimide $p$-tosylate (CMCT) at $37{ }^{\circ} \mathrm{C}$ for $20 \mathrm{~min}$ in $50 \mu \mathrm{l}$ of CMCT buffer (50 mM bicine, $\mathrm{pH}$ 8.0, $7 \mathrm{M}$ urea and $4 \mathrm{mM}$ EDTA) containing $170 \mathrm{mM} \mathrm{CMCT}$ reagent. To remove CMCT groups, the CMCT-treated RNA was subjected to alkali hydrolysis with $80 \mu \mathrm{l}$ of $\mathrm{Na}_{2} \mathrm{CO}_{3}(50 \mathrm{mM}, \mathrm{pH} 10.4)$ at $37{ }^{\circ} \mathrm{C}$ for $2 \mathrm{~h}$. One control experiment was done without CMCT treatment, and another one without alkali hydrolysis. The treated RNA ( 25 to $50 \mu \mathrm{g}$ ) was used as template in primer extension analysis with the 3'endLmjU2 oligonucleotide. In this method, the reverse transcriptase stops $1 \mathrm{nt}$ before the modified base.

\section{Plasmid constructs}

To obtain the plasmids used in this work, first a 429-bp fragment containing the U2 snRNA gene and $292 \mathrm{nt}$ from its 5'-flanking sequence, was amplified by PCR with primers 5'LmjtRNAala (5'-TGA AAA AGT TGG AGA AGT TG-3') and 3'U2Lmjend (5'-TCC CCT GGA AAC GTG GAA CT-3'). The PCR product was cloned into the pGEM-T vector (Promega) to obtain plasmid ptRNAU2. This construct was used as template in a site-directed mutagenesis assay in which a 15-bp tag sequence was inserted between positions 83 and 84 of the U2 snRNA gene with oligonucleotides 5'U2oligoTag (5'-GCC TTC GGG CAA AGG TCT GAC CTC GAC TCT AAT AGA AAT TAC AAC-3') and 3'U2oligoTag (5'-GTT GTA ATT TCT ATT AGA GTC GAG GTC AGA CCT TTG CCC GAA GGC3 '). The resultant plasmid was called ptRNAU2M. This construct lacked the $\mathrm{T}$ tract located downstream of the U2 gene. Thus, to add the transcription termination signal, a 473-bp DNA fragment from ptRNAU2M was amplified with oligonucleotides LmjtRNAala-XmaI-F (5'ACC CGG GTG AAA AAG TTG GAG AAG TTG-3') and endLmjU2-XbaI-R (5'-ATC TAG AGA AAA AAG 
GAG TTG CTC CCC TGG AAA CGT G-3') and cloned into the pGEM-T Easy vector (Promega), generating construct pComp. To obtain the vectors that contain sequential deletions of the U2 snRNA 5'-flanking sequence, several DNA fragments were amplified from pComp. The same reverse oligonucleotide (endLmjU2XbaI-R) was used in all PCR reactions, together with the following forward oligonucleotides: U2tRNA-likeXmaI-F (5'-ACC CGG GAG GAA AAG ATG CTT TCG ACG AG-3') was used for p $\Delta$ tRNA-Ala; LmjU2DcajaBXmaI-F (5'-ACC CGG GAT CCC GCC CTG GTC TCC AAA-3') was used for p $\Delta$ Box-B; LmjU2DcajaA-XmaI-F (5'-ACC CGG GGT AAG CGG CAC GGC AGT GAG-3') was employed for $\mathrm{p} \triangle \mathrm{B}$ ox-A; and primer U2Sa-XmaI-F (5'ACC CGG GAT ATC TTC TCG GCT ATT TAG C-3') was used for $\mathrm{p} \Delta-293 /-1$. All amplicons were cloned into the pGEM-T Easy vector (Promega). To obtain plasmids with base substitutions in the coding and 5'-flanking regions of the U2 snRNA, construct pComp was used as template in mutagenesis reactions performed with the following oligonucleotides: 5U2LmjmutFw (5'-TGG TAC TAA CAT ATC CAT GGA ACT ATT TAG CTA AGA T3') and 5U2LmjmutRv (5'-ATC TTA GCT AAA TAG TTC CAT GGA TAT GTT AGT ACC A-3') for pBS + 6/ +12; primers TLBTWU2LmjmutFw (5'-CAG GTA CCG TTT GGA TAG GAT CGC CCG ATT CCT TCG GGG TT-3') and TLBTWU2LmjmutRv (5'-AAC CCC GAA GGA ATC GGG CGA TCC TAT CCA AAC GGT ACC TG-3') for pBS-128/-138; oligonucleotides BalaU2LmjmutFw (5'-ATG CGG TAG GTA TTG TTG GAC GTA CCC AAC TTC TCC A-3') and BalaU2LmjmutRv (5'TGG AGA AGT TGG GTA CGT CCA ACA ATA CCT ACC GCA T-3') for pBS-263/-269; primers AalaU2LmjmutFw (5'-ACC GCG TCG GGG ATG GAT ATC ATA GCC TAG AGC GAC CGC TTA-3') and AalaU2LmjmutRv (5'-TAA GCG GTC GCT CTA GGC TAT GAT ATC CAT CCC CGA CGC GGT-3') for pBS-219/-230; oligonucleotides BlikeU2LmjmutFw (5'-GCG GGA TTC CTT CGG TTG GAC GAC CTC GTC GAA AGC A-3') and BlikeU2LmjmutRv (5'-TGC TTT CGA CGA GGT CGT CCA ACC GAA GGA ATC CCG C-3') for pBS150/-156; and primers AlikeU2LmjmutFw (5'-CGC GGC GTT TTT TTT GAT ACC ATC GCC TAC CGT TTG GAG ACC-3') and AlikeU2LmjmutRv (5'-GGT CTC CAA ACG GTA GGC GAT GGT ATC AAA AAA AAC GCC GCG-3') for pBS-105/-116. Finally, to generate pDBS that possesses a double mutation, plasmid pBS105/-116 was used as template in a mutagenesis reaction with oligonucleotides BlikeU2LmjmutFw and BlikeU2LmjmutRv. All mutagenesis reactions were performed with a QuikChange Lightning Site-Directed Mutagenesis Kit (Agilent Technologies, Santa Clara, USA). In boxes A and B, the mutated nucleotides correspond to the most conserved positions, which have been shown to be important for Pol III transcription in other organisms $[32,33]$. The identity of each insert was confirmed by sequencing using the T7 and SP6 oligonucleotides.

\section{Real-time quantitative PCR}

Around $1 \mu \mathrm{g}$ of DNase I-treated RNA from transiently transfected cells (from three independent transfections) was used as template for first strand cDNA synthesis using SuperScript ${ }^{\text {ti }}$ III Reverse Transcriptase (Invitrogen) and 2 pmol of oligonucleotides 3'U2Lmjend or Alfa-tub3' (5'-GTA GTT GAT GCC GCA CTT GAA G-3'). The cDNA was analyzed by real-time quantitative PCR (RTqPCR) assays using the Platinum SYBR Green qPCR SuperMix-UDG kit (Invitrogen) in a Rotor-Gene 3000 cycler (Corbett Research, Mortlake, Australia) according to the manufacturer's recommendations. All RT-qPCR reactions were performed at least in duplicate, using primers and conditions that were optimized to produce a single amplicon of the correct size. Each amplification product was analyzed for specificity by both agarose gel electrophoresis and melt curve analysis. Standard curves for primer pairs were derived from genomic and plasmid DNA dilution series and ranged in their $\mathrm{r}^{2}$ value from 0.98 to 1.0 . PCR efficiencies were near to $100 \%$ for the used genes, so the data were analyzed by the $2^{-\Delta \Delta C q}$ method. For normalization of the data we used $\alpha$ tubulin (LmjF.13.0280) as a reference gene, and all values were represented relative to data from cells transfected with plasmid pComp. The tagged-U2 snRNA was amplified with primers U2Sa-5' and LmjU2tag-Rev. The $\alpha$-tubulin transcript was amplified with oligonucleotides Alfa-tub-5' (5'-AGA AGT CCA AGC TCG GCT ACA C-3') and a-tubLmjrvs (5'-GGT CGT AGA TGG CCT CAT TG-3'). To analyze RNA from cells transfected with $\mathrm{pBS}+6 /+12$, the same conditions indicated above were performed, but using $\mathrm{U} 2 \mathrm{qFw} 2$ as the sense primer in the PCR assays.

\section{Northern blot analysis}

Total RNA $(10-30 \quad \mu \mathrm{g})$ was separated on $10 \%$ polyacrylamide-8 M urea gels. After electrophoresis, RNA was transferred to Hybond $\mathrm{N}+$ membranes (GE Healthcare, Little Chalfont, England) by electro-blotting using a Trans-Blot SD Semi-Dry Transfer Cell (Bio-Rad, Hercules, USA). The tRNA-Ala probe corresponds to the Ala-Rvs (5'-TCG ATC CCA ATA CCT ACC GC-3') oligonucleotide, the tRNA-like probe corresponds to the 5'LmjU2DcajaB oligonucleotide, the U2 snRNA probe to the 3'endLmjU2 oligonucleotide, and the tag probe to the LmjU2tag-Rev oligonucleotide. Primers were labelled with $\left[\gamma_{-}{ }^{32} \mathrm{P}\right] \mathrm{ATP}$ using T4 kinase. Hybridizations were performed in $6 \times$ SSPE $\left(60 \mathrm{mM} \mathrm{Na}_{2} \mathrm{HPO}_{4}, 0.9 \mathrm{M} \mathrm{NaCl}\right.$ and $6 \mathrm{mM}$ EDTA), $5 \times$ Denhardt's reagent and $1 \%$ SDS 
at $42{ }^{\circ} \mathrm{C}$. Washing was carried out at $55^{\circ} \mathrm{C}$ in $0.2 \times \mathrm{SSC}$ and $0.1 \%$ SDS.

\section{Results}

Genomic context of the U2 snRNA in L. major and other trypanosomatids

The L. major genome contains a single copy of the U2 snRNA gene (LmjF.31.snRNA.01), which is embedded into an RNAP II polycistronic unit on chromosome 31 [34]. The U2 snRNA gene is flanked by two different amino acid transporter genes: LmjF.31.0580 and LmjF.31.0570 (Fig. 1a). A divergently-oriented tRNA-Ala gene (LmjF.31.
TRNAALA.01) is located $211 \mathrm{bp}$ upstream of the U2 snRNA gene (Fig. 1a). To determine the genomic context of U2 snRNA genes in other species of Leishmania, we examined the genome databases of $L$. donovani (strain BPK282A1) [35], L. infantum (JPCM5), L. braziliensis (M2904) [36, 37], L. mexicana (U1103) [37] and L. tarentolae (Parrot-TarII) [38]. Similarly to L. major, in all of the Leishmania species analyzed the U2 snRNA is encoded by a single-copy gene inserted into the same polycistronic unit (Fig. 1a), which is located on chromosome 31 in $L$. donovani, L. infantum, L. braziliensis and L. tarentolae, but on chromosome 30 in L. mexicana (as chromosomes

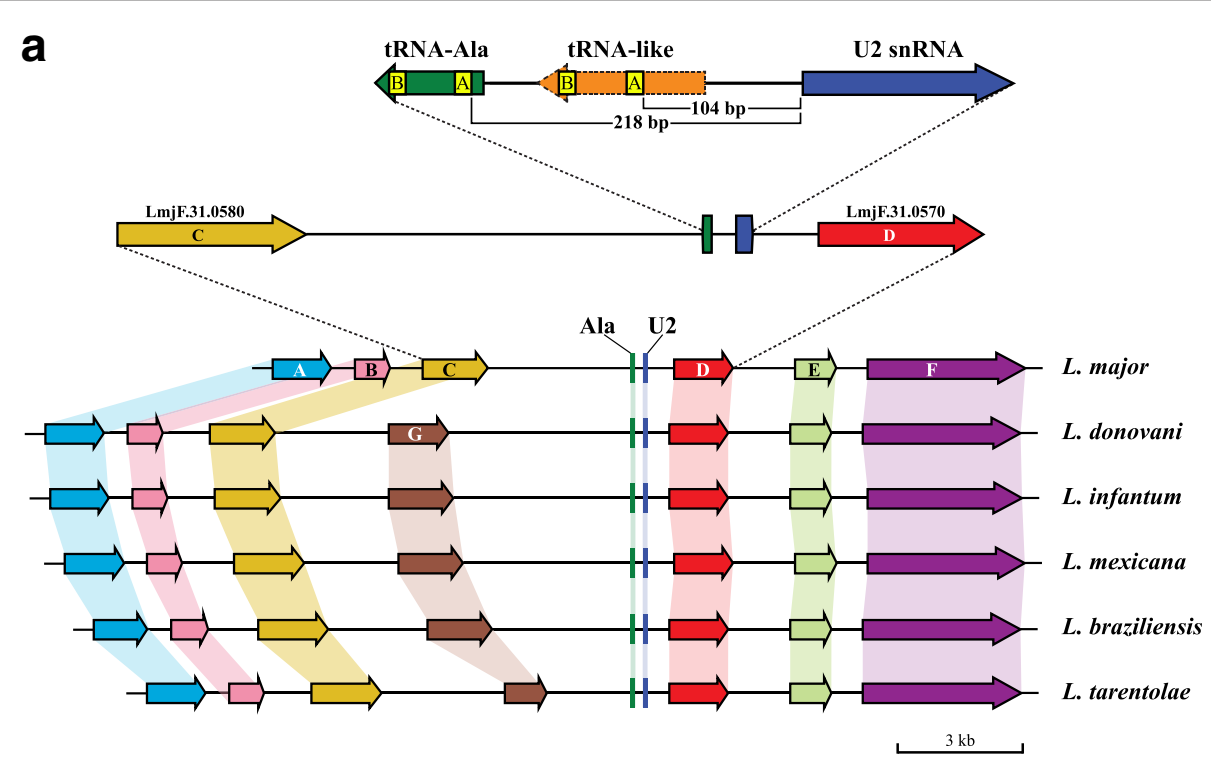

b

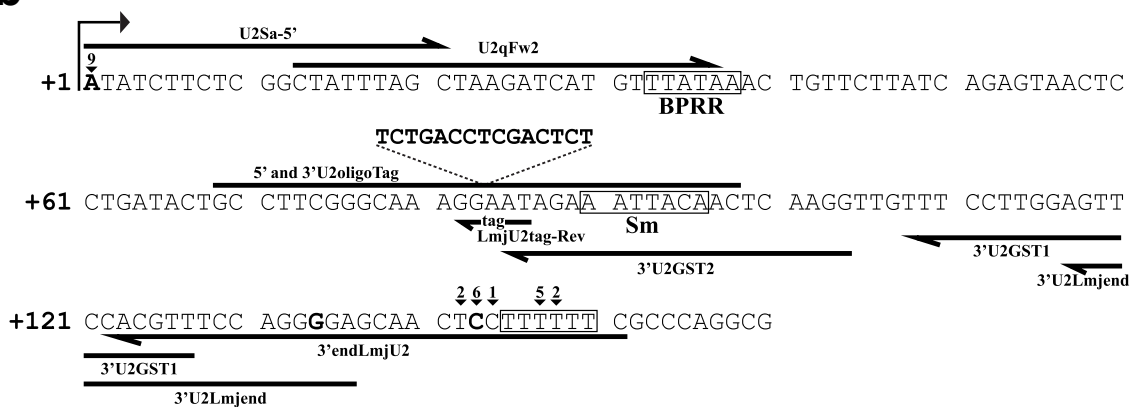

Fig. 1 Genomic context and sequence of the U2 snRNA gene in L. major. a Synteny of U2 snRNA genes in different species of Leishmania. Orthologues genes are joined by colored lines. In L. major, gene A (light blue) corresponds to LmjF.31.0600, gene B (pink) to LmjF.31.0590, gene C (mustard) to LmjF.31.0580, gene D (red) to LmjF.31.0570, gene E (light green) to LmjF.31.0560 and gene $F$ (purple) to LmjF.31.0550. Gene G (brown) is absent in L. major, but corresponds to LdBPK_310600.1 in L. donovani. Two enlargements of the L. major U2 snRNA gene are shown. On the top scheme are presented the U2 snRNA (blue), the tRNA-like (orange) and tRNA-Ala (green) genes. Boxes A and B within the tRNA-like and tRNA-Ala gene are shown in yellow. Distances between the U2 snRNA gene and boxes A from the tRNA-like (104 bp) and the tRNA-Ala gene (218 bp) are indicated. Figure is drawn to scale. $\mathbf{b}$ Sequence of the L. major U2 snRNA gene and 3 '-flanking region. The A residue shown in bold type (position +1 ) corresponds to the TSS mapped by primer extension and 5'-RACE analyses (the arrowhead specify the number of clones found at that position). At the $3^{\prime}$ end, the cluster of T nucleotides is shown in a box. The 3' termini mapped by RT-PCR are denoted with arrowheads, specifying the number of clones found at each position. The mature $3^{\prime}$ terminus of the U2 snRNA ( $C$ at position +143) is shown in bold type. The missanotated 3' end of U2 at position $134(\mathrm{G})$ is also shown in bold type. The locations of putative BPRR and Sm site are shown. The sequence of the tag, inserted between positions $83(\mathrm{G})$ and $84(\mathrm{~A})$, is also indicated. The sequence and location of some of the oligonucleotides used in this study are shown. To facilitate the analysis of the results, the sequence is shown as the reverse-complement of the annotated sequence of chromosome 31 from the TriTrypDB database 
8 and 29 are fused in this species). Moreover, the divergently oriented tRNA-Ala gene is present in all of the species analyzed. Thus, synteny of the U2 snRNA locus is observed among Leishmania species. Interestingly, the genome of $L$. major lacks an extra amino acid transporter gene (Fig. 1a, gene G) that is present in all other species of Leishmania.

To extend the analysis of gene copy number of the U2 snRNA to other trypanosomatids, the genomic databases of members of the genera Trypanosoma, Crithidia, Endotrypanum and Leptomonas were examined. A single copy of the U2 snRNA gene is present in T. brucei (strains Lister 427, DAL972 and TREU927), T. evansi (STIB 805), T. vivax (Y486), C. fasciculata (Cf-Cl), E. monterogeii (LV88), L. pyrrhocoris (H10) and L. seymouri (ATCC 30220). Interestingly, two different strains of $T$. cruzi (CL Brener and Dm28_c) have three copies of the U2 snRNA gene. Thus, trypanosomatids possess only one copy of the U2 snRNA gene, with the exception of T. cruzi that contains three copies of this gene.

\section{Mapping of transcription initiation and termination sites in the U2 snRNA}

According to the TriTrypDB databases, the length of the L. major U2 snRNA gene is 134 bp (Fig. 1b). However, sequence comparisons (Additional file 1: Figure S1) suggest that the $3^{\prime}$ end of the gene is missanotated. Thus, to determine the correct size of the U2 snRNA we mapped both the $5^{\prime}$ and 3 ' termini of this snRNA. First, to locate the TSS, a primer extension assay was carried out. As shown in Fig. 2a, the TSS of U2 snRNA corresponds to an A residue ( $\mathrm{T}$ in the complementary sequence), which is equivalent to the TSS previously identified in T. cruzi, T. brucei and L. collosoma $[39,40]$. To corroborate the location of the TSS in the L. major U2 snRNA, a 5'RACE assay was conducted. Analysis of nine cloned sequences confirmed that transcription of the U2 snRNA initiates at the A nucleotide (Fig. 1b). Thus, the TSS of the U2 snRNA is conserved in trypanosomatids.

To determine transcription termination sites of $\mathrm{U} 2$ snRNA in L. major, RT-PCR was performed with total RNA that was poly(A)-tailed in vitro. Transcription of U2 snRNA was found to end within a tract of six Ts located from 145 to $150 \mathrm{bp}$ downstream of the TSS (Fig. 1b). In two of the clones analyzed, the poly(A) tail was added after the fourth $\mathrm{T}$, and in five clones it was added after the third $\mathrm{T}$ residue. Trimming of these transcripts generate shorter RNA molecules, as one clone ended at nucleotide $+144(\mathrm{C})$, six clones ended at position $+143(\mathrm{C})$, and two clones at base $+142(\mathrm{U})$. Thus, it appears that during the processing of the 3 ' end of the U2 snRNA there are several populations of RNA with different 3 ' ends. Since most clones ended at the $\mathrm{C}$ residue located at position +143 , we considered this nucleotide as the mature 3 terminus of the U2 snRNA in $L$. major (Fig. 1b). Consequently, by mapping both $5^{\prime}$ and 3 ' ends of U2 snRNA in L. major, we have determined that the mature transcript is $143 \mathrm{nt}$ long.

\section{Localization of pseudouridine residues in the U2 snRNA}

Pseudouridylation of the U2 snRNA is essential for snRNP assembly and splicing in vertebrates and yeast [9]. To determine the presence of pseudouridine residues in the L. major U2 snRNA, total RNA was treated with CMCT followed by alkaline treatment and primer extension analysis. Considering that in this procedure the reverse transcriptase extension stops one base downstream of the CMCT-modified nucleotide, we found six pseudouridines in the U2 snRNA sequence at positions 14, 34, 36, 55, 86 and 93 (Fig. 2b, Lane 6). A control experiment was done without $\mathrm{CMCT}$ treatment to detect natural pauses of the reverse transcriptase, probably as a result of RNA degradation or the presence of stable RNA secondary structures (Fig. 2b, Lane 5). Another control without alkali hydrolysis was carried out to identify all the positions where the CMCT reagent was attached (Fig. 2b, Lane 7). In the experiment shown in Fig. $2 b$, the band that corresponds to pseudouridine at position 14 is very faint. However, in other experiments this band was clearly observed (Fig. 2c, Lane 6).

To determine if the pseudouridines are located in important functional domains of the U2 snRNA, the predicted secondary structure of the RNA molecule was generated and putative branch point recognition region (BPRR) and Sm binding sites were identified (Fig. 3a). Regarding the BPRR, the well-conserved metazoan sequence GUAGUA is replaced by UUAUAA in the $L$. major U2 snRNA (Fig. 3a). Also, the Sm site in the U2 snRNA, previously identified as AAUUACA (from nucleotides 90 to 96) (Fig. 3a), differs from the metazoan consensus sequence $\mathrm{RAU}_{3-6} \mathrm{GR}$ [41]. Interestingly, pseudouridines at positions 34 and 36 are located within the BPRR, and pseudouridine at base 93 is situated in the Sm site (Fig. 3a). It is worth noting that a pseudouridine in the $\mathrm{Sm}$ site has not been reported in any other organism.

As expected, the sequence of the U2 snRNA is conserved across trypanosomatids, as shown by a multiple sequence alignment (Additional file 1: Figure S1) and a sequence logo created with the WebLogo application [42] (Fig. 3b). Homology between the L. major U2 snRNA and its orthologues in other species of Leishmania is very high, ranging from $95.1 \%$ identity with $L$. braziliensis to $98.6 \%$ identity with $L$. tarentolae. Also, $L$. major U2 snRNA shows $69.4 \%$ and $76.2 \%$ identity to its orthologues in T. cruzi and T. brucei, respectively. Notably, we found that the U2 snRNA genes from all of the 21 species of trypanosomatids that we analyzed 

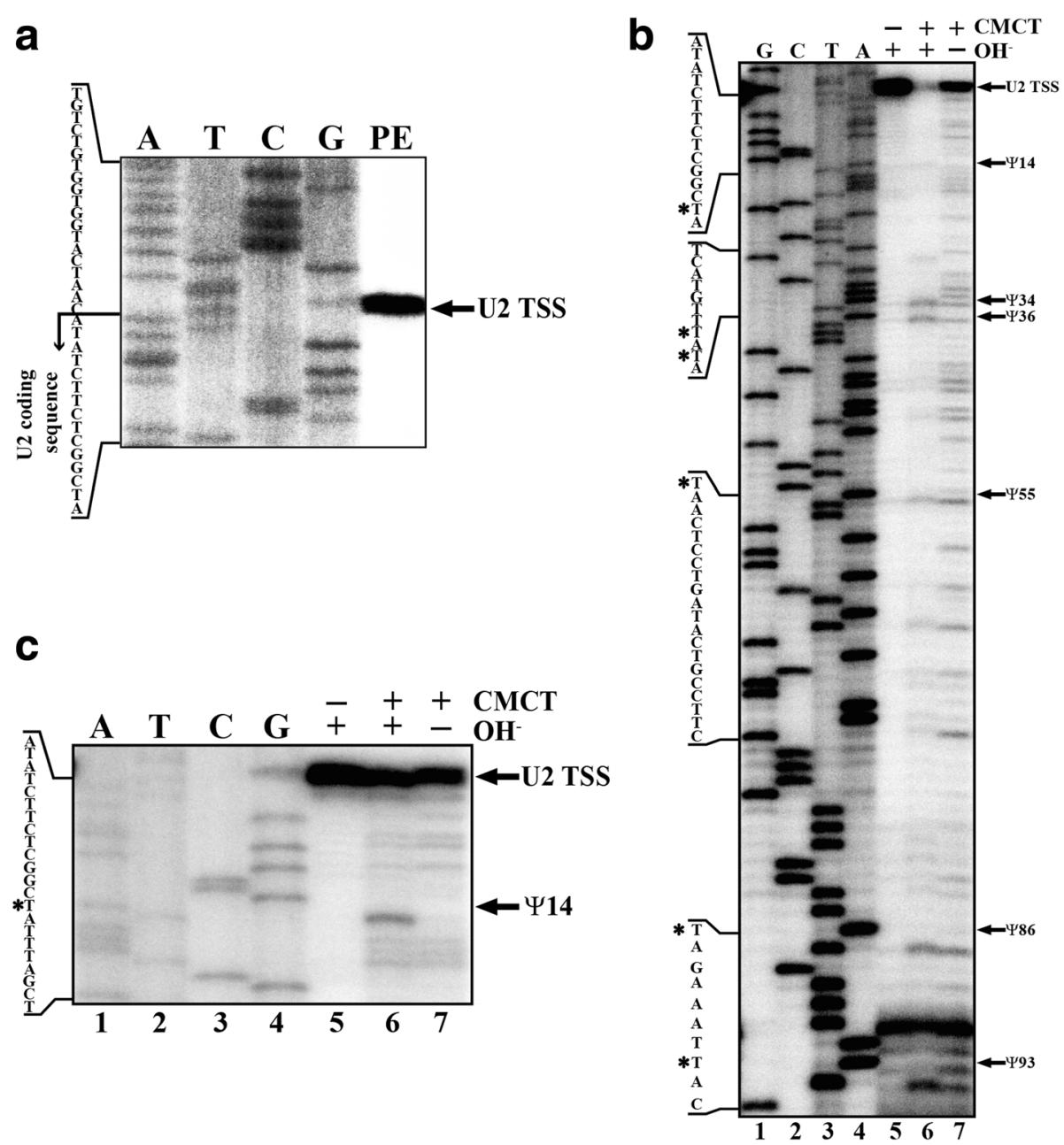

Fig. 2 Primer extension analyses of the L. major U2 snRNA. a Mapping of the TSS of U2 snRNA in wild type promastigotes of L. major. Total RNA was subjected to primer extension with oligonucleotide 3'endLmjU2, and the products were separated on an $8 \%$ polyacrylamide denaturing gel (Lane PE), along with a sequence ladder obtained with the same oligonucleotide (Lanes A, T, C and G). The arrow indicates the TSS, which corresponds to an A residue. Because the sequencing reactions were performed with 7-deaza dGTP (and the primer extension with dGTP), there is a size difference of about 1 bp between the sequence ladder and the primer extension product [48]. b Mapping of pseudouridines in U2 snRNA of L. major. Primer extension reactions were performed on total L. major RNA treated (+) or not (-) with CMCT for 20 min at $37{ }^{\circ} \mathrm{C}$. CMCTtreated RNA was subjected to alkali hydrolysis $(\mathrm{OH},+)$. One control experiment was done without alkali hydrolysis $(\mathrm{OH},-)$. Primer extension and sequencing reactions were performed with oligonucleotide $3^{\prime}$ endLmjU2. Arrows indicate the identified pseudouridines $(\Psi)$ (reverse transcriptase stops 1 base before the pseudouridine). A partial sequence is shown on the left, indicating with asterisks the positions of the pseudouridylated nucleotides. c Primer extension-based pseudouridylation assay carried out as described in panel b. Only the upper part of the gel is shown

contain a $\mathrm{T}$ residue ( $\mathrm{U}$ in the corresponding RNA sequence) in five of the six pseudouridylated positions identified in the L. major U2 snRNA (Fig. $3 \mathrm{~b}$ and Additional file 1: Figure S1). The exception is position 93, which corresponds to a $\mathrm{C}$ or a $\mathrm{G}$ in five of the species analyzed. Thus, potentially pseudouridylated nucleotides are conserved in trypanosomatids.

\section{Characterization of the promoter elements in the L. major U2 snRNA}

In T. brucei and L. collosoma, snRNA promoters consist of boxes A and B located inside tRNA or tRNA-like sequences found in the opposite direction of the snRNA.
Interestingly, box A is consistently located 104 to $106 \mathrm{bp}$ upstream of the snRNA. In L. major, box A of the tRNA-Ala gene is located 218 bp upstream of the U2 snRNA (Fig. 1a). However, we identified a tRNA-like sequence between the tRNA-Ala and the U2 snRNA genes (Fig. 1a). Boxes A and B from the tRNA-like sequence were located 105 and $150 \mathrm{bp}$ upstream of the U2 snRNA, respectively. Thus, boxes A and B from the tRNA-like might control the transcription of the U2 snRNA. To test this hypothesis, we analyzed the transcription of several plasmid constructs after transient transfection of promastigotes. To identify transcripts produced by the transfected plasmids we marked the 


\section{a}
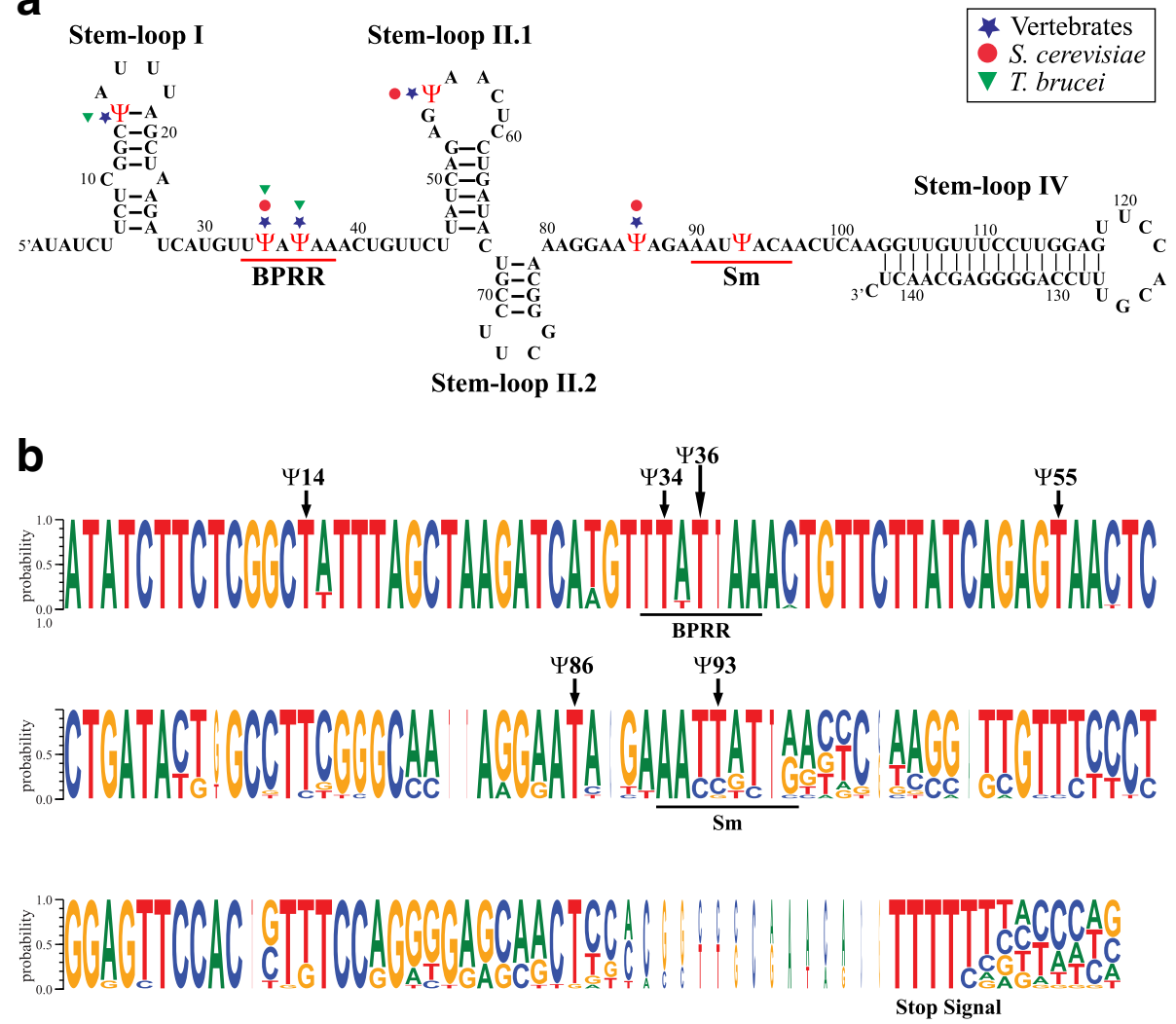

Fig. 3 Sequence conservation and secondary structure of the U2 snRNA. a Predicted secondary structure of the L. major U2 snRNA. Stem-loops I, II.1, II.2 and IV are indicated. Also, BPRR and Sm site are shown. Pseudouridine residues (red $\psi$ ) identified in this study are denoted. Pseudouridines conserved between L. major and vertebrates (blue star), S. cerevisiae (red circle) and T. brucei (green triangle) are also indicated. b Consensus sequence of the U2 snRNA gene in trypanosomatids. The sequences of the U2 snRNA genes and 3'-flaking regions from 21 species of trypanosomatids (see Additional file 1: Figure S1) were aligned with the DNAMAN software. Using the WebLogo application, the generated pattern was represented as a sequence logo by residue probabilities. BPRR, Sm site and stop signal are shown. The positions of the pseudouridine residues ( $\Psi)$ mapped in L. major are indicated with arrows

external U2 snRNA gene by insertion of a 15-nt tag sequence between positions +83 and +84 (Figs. $1 \mathrm{~b}$ and $4 \mathrm{a}$ ). Since no promoter sequences have been reported in this region, it is unlikely that the tag sequence could compromise the activity of a potential internal promoter element. Indeed, expression of tagged U2 snRNA was demonstrated by Northern blot (Additional file 2: Figure S2).

Thus, total RNA was extracted $24 \mathrm{~h}$ post-transfection and primer extension analyses were performed using an oligonucleotide complementary to the tag. As shown in Fig. 4b, with pComp (plasmid 1), which contains the complete $5^{\prime}$ upstream region (tRNA-Ala and tRNA-like sequences) two primer extension products were detected (Fig. 4b, Lane 1). The longest product corresponds to the U2 snRNA TSS, while the other product was mapped to an A located at position +3 (marked with an asterisk in Fig. $4 \mathrm{~b}$ ) that might represent a reverse transcriptase stop. As expected, these bands were not present in the mock-transfected cells and cells transfected with a control vector (Fig. 4b, Mock and pLMRIB). Deletion of the tRNA-Ala gene (p $\Delta$ tRNA-Ala) did not affect the abundance of the U2 snRNA bands (Fig. 4b, Lane 2). In contrast, elimination of box $\mathrm{B}$ from the tRNA-like ( $\mathrm{p} \Delta \mathrm{Box}-\mathrm{B}$, Lane 3), deletion of box A from the tRNA-like ( $\mathrm{p} \Delta \mathrm{Box}-\mathrm{A}$, Lane 4$)$ and removal of the entire 5 flanking region $(\mathrm{p} \Delta$ 293/-1, Lane 5) reduced the U2 snRNA transcripts to undetectable levels (Fig. 4b). Therefore, these results show that sequences within the tRNA-like are needed for transcription of the L. major U2 snRNA. To evaluate the presence of a possible internal promoter element in the snRNA gene, the sequence of the first nucleotides of the gene (from +6 to +12 ) were mutated (Additional file 3: Figure S3). In the primer extension analysis with this vector $(\mathrm{pBS}+6 /+12$, Lane 6$)$ the snRNA transcripts were strongly reduced, showing that the first bases of the U2 snRNA gene are required for expression of the gene. In contrast, mutation of the sequence located between boxes $\mathrm{A}$ and $\mathrm{B}$ in the tRNA-like did not affect the abundance of the U2 snRNA (pBS-128/-138, Lane 7) (Fig. 4b). A nonspecific product that was observed in all the lanes was 


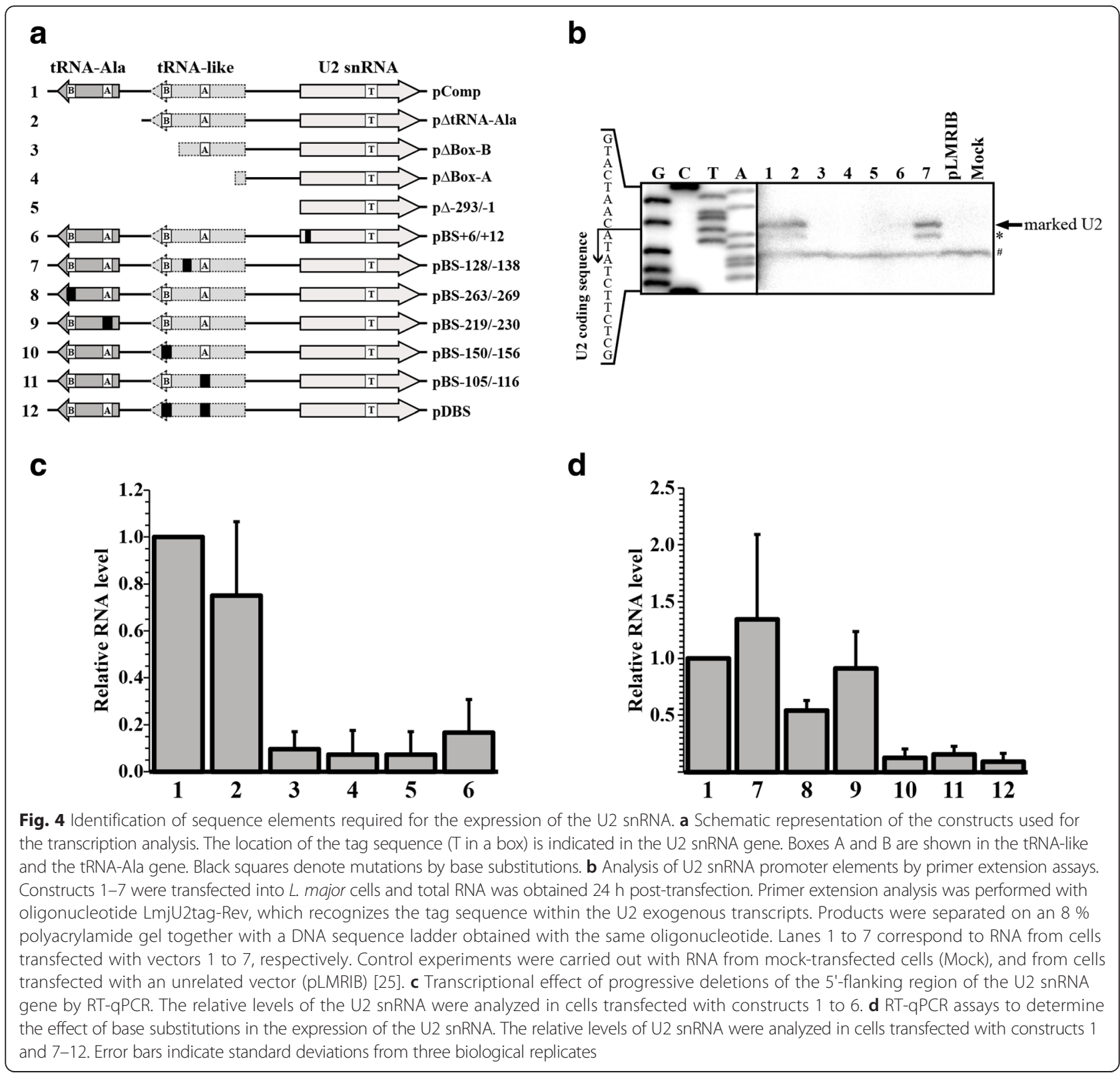

used as a control for RNA recovery (marked with a hash character in Fig. 4b).

To obtain a more precise determination of the abundance of the U2 snRNA transcripts, RNA from transientlytransfected cells was analyzed by RT-qPCR. As in the primer extension analysis, to discriminate between endogenous U2 and U2-tagged transcripts, an oligonucleotide complementary to the tag sequence was used. As shown in Fig. 4c, the U2 snRNA relative levels were severely reduced (by 90-93\%) when box B of the tRNA-like and sequences downstream were deleted (compare bar 1 to 3-5). Thus, these results are in agreement with the primer extension results (Fig. 4b, Lanes 3-5), and indicate that the tRNA-like is needed for the in vivo expression of the U2 snRNA. To analyze independently the transcriptional roles of boxes $\mathrm{A}$ and $\mathrm{B}$ from the tRNA-like, base substitutions were produced in these two elements (Additional file 3: Figure S3). We observed that mutations in box B (Fig. 4d, bar 10), box A (Fig. 4d, bar 11) and both boxes (Fig. 4d, bar 12) drastically diminished the U2 snRNA levels to 13,16 and $9 \%$, respectively. By contrast, a slight increase in the levels of U2 snRNA was found with the control vector that bears a mutation in the region located between boxes A and B in the tRNA-like, where no promoter element is found (Fig. 4d, bar 7). In agreement with the primer extension analysis, mutation of the first nucleotides of the U2 snRNA gene diminished the RNA levels to $17 \%$ of the value of the control (Fig. 4c, bar 6). 
An unexpected result was that deletion of the tRNAAla gene, located upstream of the tRNA-like, produced a $25 \%$ reduction in the levels of the U2 snRNA (Fig. 4c, bar 2). To further explore this effect, base substitutions in boxes A and B of the tRNA-Ala gene were generated and analyzed. Interestingly, when box B was mutated, the expression of the U2 snRNA was reduced to $54 \%$ of the value for the control (Fig. 4d, bar 8). In contrast, mutations in box A from the tRNA-Ala gene have a slight impact on the abundance of the U2 snRNA (Fig. 4d, bar 9). Therefore, our results show that box B from the tRNA-Ala gene located upstream of the tRNAlike is important for transcription of the U2 snRNA in $L$. major.

\section{The tRNA-like sequence associated to the U2 snRNA is transcribed}

To determine whether the tRNA-like sequence linked to the L. major U2 snRNA gene is transcribed, a Northern blot experiment was performed with total RNA isolated from wild type L. major promastigotes. A band of around $109 \mathrm{nt}$ was detected, indicating that the tRNA-like is transcribed in L. major (Fig. 5a, middle panel). Several larger and weaker bands were also observed, which may represent longer tRNA-like transcripts or unspecific bands. Control experiments to detect the U2 snRNA and the tRNA-Ala showed the expected bands of around 143 and $73 \mathrm{nt}$, respectively (Fig. 5a). Densitometry analysis of the observed bands showed that the tRNA-like is at least 10fold less abundant than the U2 snRNA.

The TSS of the tRNA-like was mapped by primer extension (Fig. 5b). The most abundant band corresponded to a $C$ residue ( $G$ in the complementary sequence) (labelled with a red asterisk in Fig. 5b) located 65 bases upstream of the U2 snRNA (Fig. 5c). If we assume that transcription of the tRNA-like ends in the T-tract located downstream of the gene (Fig. $5 \mathrm{c}$ ), the tRNA-like transcript would be 109 nt long, which is in agreement with the size observed in the Northern blot (Fig. 5a). The smaller bands detected in the primer extension

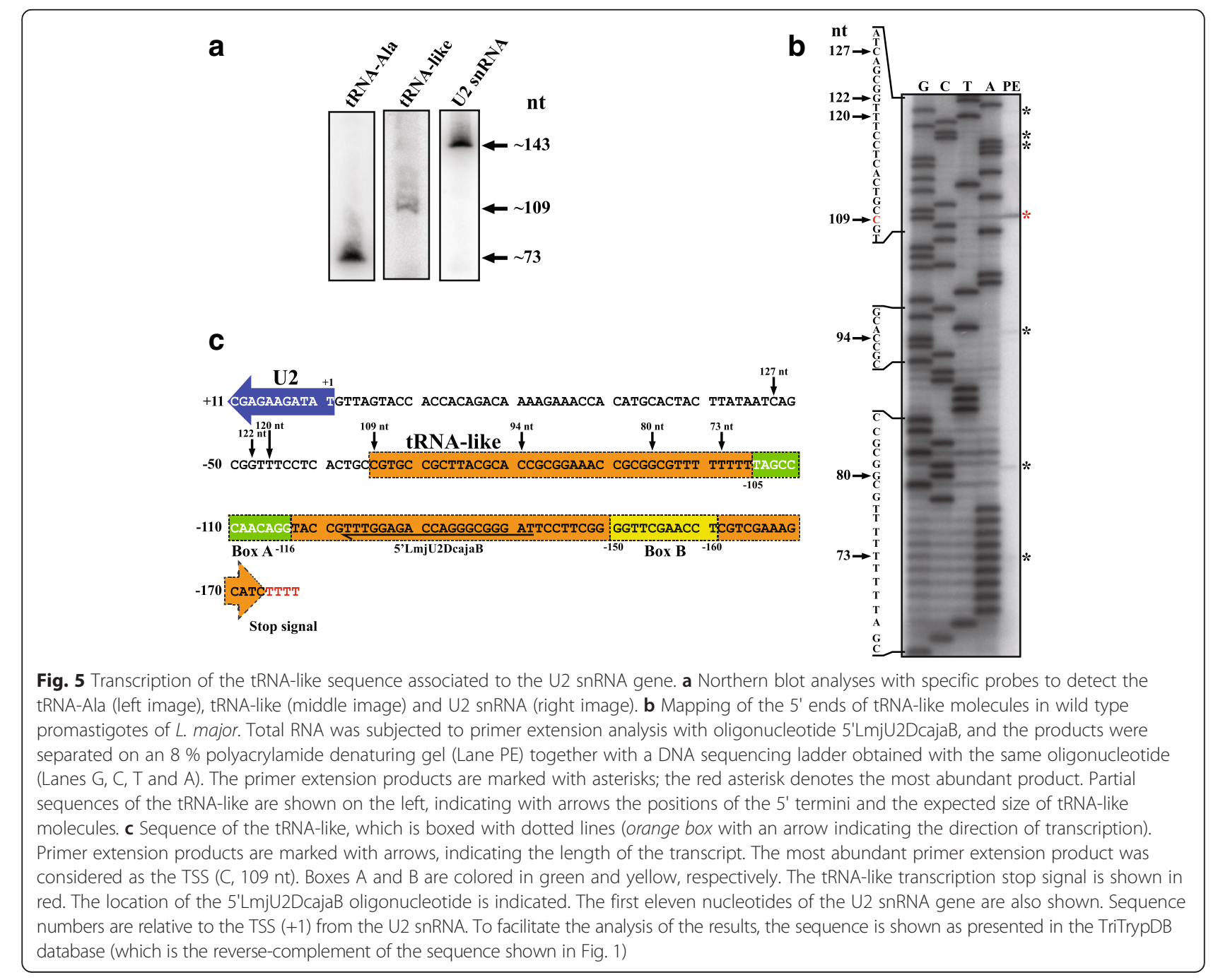


analysis (73, 80 and $94 \mathrm{nt})$ might represent reverse transcriptase stops, while the larger bands (120, 122 and $127 \mathrm{nt}$ ) could denote alternative TSS (black asterisks in Fig. 5b, and black arrows in Fig. 5c).

\section{Sequence analysis of boxes $A$ and $B$ from tRNA-like sequences associated to U2 snRNA genes}

Sequence analysis of boxes A (TAG CCC AAC AGG) and B (GGT TCG AAC CT) from the tRNA-like associated to the $L$. major $\mathrm{U} 2$ snRNA revealed that they are similar to boxes A (TAG CTC AGA TGG) and B (GGA TCG ATA CC) from the tRNA-Ala gene. To compare these sequence elements across trypanosomatids, we first searched for boxes A and B in tRNA-like sequences associated to U2 snRNA genes in the genome databases of 18 additional species of trypanosomatids (Fig. 6a). A tRNA-like sequence with boxes A and B was found in all the species analyzed (Fig. 6a); and sequence logos were obtained for both sequence elements (Fig. 6b, upper logos). For comparison, sequences of boxes $\mathrm{A}$ and $\mathrm{B}$ from all of the 258 classic tRNAs found in TriTryps [43] were aligned and logo sequences were generated (Fig. 6b, lower logos). Regarding tRNA-like sequences, analysis of the sequence logo from box A showed a high conservation of most bases, with the exception of the position labelled with an asterisk (which is absent in most species analyzed) and base 9 (Fig. 6b, upper logo). Box B is even more conserved, as positions 1 to 5 (GGTTC) are present in all the sequences, and the rest of the bases are conserved, with the exception of positions 8 and 11 (Fig. 6b, upper logo). In general, high sequence conservation was observed when comparing boxes $B$ from tRNA-like sequences and classic tRNA genes, since position 11 is the only one that is not conserved (Fig. 6b, right logos). On the other hand, boxes $\mathrm{A}$ are not as highly conserved as boxes $\mathrm{B}$, since positions $5,6,7,9,11$ and $(*)$ differ between tRNA-like and classic tRNA genes (Fig. 6b, left logos).

It is interesting to note that a distance of $104 \mathrm{bp}$ separates the U2 snRNA gene and box A from the tRNA-like sequence in most trypanosomatids (Fig. 6a). Moreover, the distance that separates box A from box B in the tRNA-like sequences is conserved in Leishmania, fluctuating between 33 bases (in L. major and L. braziliensis) and 36 bases (in L. mexicana). In contrast, this distance is not conserved in tRNA-like sequences from trypanosomes, as it varies from 30 bases (T. vivax) to 44 bases (T. cruzi) (Fig. 6a). Thus, our analysis shows that, while boxes $\mathrm{A}$ are more divergent in sequence, their position in relation to the U2 snRNA gene is almost invariable. On the other hand, the sequences of boxes B are highly conserved but their distance to box A (or to the snRNA gene) is variable across trypanosomatids.

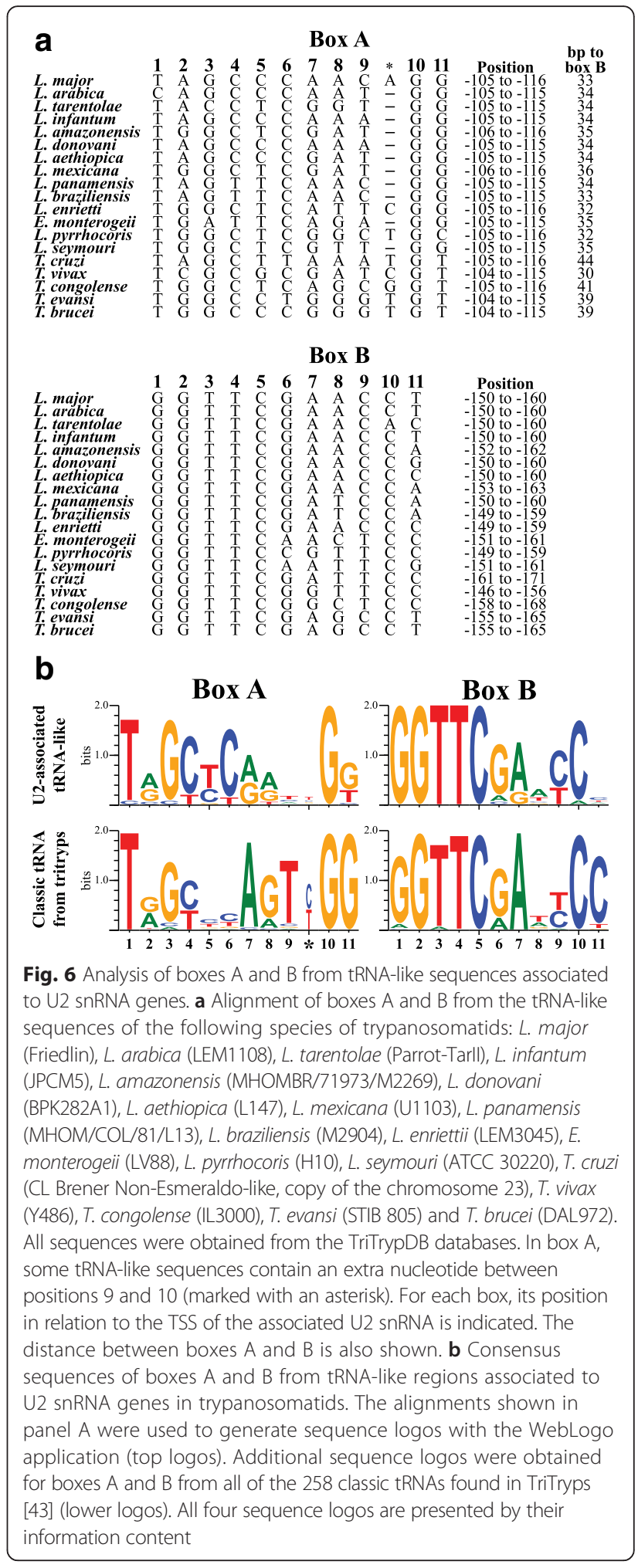

\section{Discussion}

In this work we have studied the sequence, structure and genomic context of the U2 snRNA gene in Leishmania and other trypanosomatids, and have identified 
the sequences that drive transcription of the U2 snRNA in L. major. Although in general the sequence of the U2 snRNA is highly conserved in trypanosomatids, the sequence and length of its 3 ' end are variable (Fig. $3 \mathrm{~b}$ and Additional file 1: Figure S1). Thus, we mapped both $5^{\prime}$ and 3 ' ends of U2 snRNA in L. major and determined that the mature transcript is $143 \mathrm{nt}$ long (Fig. 1b), which is similar to the 143 and $145 \mathrm{nt}$ reported for the U2 snRNA in T. cruzi and T. brucei, respectively [44, 45].

The predicted secondary structure of the U2 snRNA in L. major (Fig. 3a) resembles the structure reported for T. brucei, as they both lack stem-loop III (Fig. 3a). Out of the six pseudouridine residues that we found in the $L$. major U2 snRNA (Fig. 2b), five are also present in vertebrates (positions 14, 34, 36, 55 and 86 in L. major), three have been found in S. cerevisiae (positions 34, 55 and 86) $[8,9]$, and three are present in T. brucei (positions 14, 34 and 36) [31] (Fig. 3a). It is worth noting that the only pseudouridine that is conserved in all of the organisms analyzed is the one located at position 34 , which is part of the motif involved in the branch site recognition (Fig. 3a). Pseudouridine at position 36 is also located in the BPRR. Interestingly, the L. major U2 snRNA contains one pseudouridine that has not been reported in other organisms, which is located within the Sm site at position 93 (Fig. 3a). Thus, it is likely that the pseudouridines we mapped in the $L$. major U2 snRNA are important for snRNP assembly and trans-splicing.

A tRNA-like was found between the U2 snRNA and the tRNA-Ala genes in L. major (Fig. 1a). Primer extension and RT-qPCR analyses showed that transcription of the U2 snRNA gene is dependent on boxes A and B located inside the tRNA-like sequence, since deletion or mutation of these elements strongly reduced the level of the snRNA. Also, an intragenic promoter element was found close to the 5 ' end of the U2 snRNA gene (Fig. 4). Similar promoter elements drive the expression of the U2 snRNA gene in T. brucei [17]. Moreover, boxes A and/or B located in tRNA-like or tRNA genes have been shown to control the expression of the U1, U3 and U6 snRNA genes in T. brucei, the U4 snRNA gene in L. collosoma and 7SL RNA genes in T. brucei and L. collosoma $[18,19,22,23,46]$. However, unlike any other snRNA or 7SL RNA genes in trypanosomatids, our data show that box B from the upstream tRNA-Ala also participates in the regulation of transcription of the U2 snRNA gene in $L$. major. While mutation of box B from the tRNA-like reduced the U2 snRNA expression by $87 \%$, base substitutions in box B from the tRNA-Ala decreased the levels of the U2 snRNA by $46 \%$ (Fig. $4 \mathrm{~d}$, compare bars 8 and 10). Thus, it is possible that box B from the tRNA-Ala participates in the fine-tuning of transcription of the L. major U2 snRNA.
At present it is not known how boxes $\mathrm{A}$ and $\mathrm{B}$ from the associated tRNA or tRNA-like control transcription of the snRNA. It has been proposed that transcription factors binding to boxes $\mathrm{A}$ and $\mathrm{B}$ would be shared by the snRNA and tRNA (or tRNA-like) genes. Alternatively, boxes $\mathrm{A}$ and $\mathrm{B}$ could regulate snRNA expression by an indirect mechanism involving the binding of transcription factors to the tRNA gene, which in turn could generate changes in chromatin structure that would allow transcription of the snRNA [21]. In this regard, very little is known about RNAP III transcription factors in trypanosomatids. From the two transcription factors required for the expression of tRNA genes, TFIIIC and TFIIIB, only the latter has been identified in trypanosomatids [47]. Thus, it is possible that the highlytranscribed tRNA-Ala gene, and the tRNA-like sequence, might bind abundant transcription factors and produce an open chromatin conformation that could facilitate transcription of the U2 snRNA in L. major.

Our results showed that the tRNA-like associated to the U2 snRNA is transcribed, generating a major transcript of around $109 \mathrm{nt}$ (Fig. 5a, middle panel). Transcription of tRNA-like sequences associated with the $T$. brucei U2 snRNA and the L. collosoma U4 snRNA have also been demonstrated, producing transcripts of 89 and 90 bases, respectively [20,23]. Densitometric analysis indicated that the tRNA-like transcript is at least 10-fold less abundant than the U2 snRNA. Thus, if the U2 snRNA and the tRNA-like are transcribed together and at the same rate, the tRNA-like should be less stable than the U2 snRNA. The predicted secondary structure of the tRNA-like was very different from the expected for a tRNA, as it does not fold into the classical cloverleaf structure (Additional file 4: Figure S4). Consequently, it is possible that this atypical secondary structure might contribute to the apparent instability of the tRNA-like transcript. Other possibility is that the transcription rate in the two directions is different and, accordingly, lower amounts of tRNA-like are produced.

Our data show that all of the Leishmania species analyzed contain a single U2 snRNA gene, which is highly syntenic (Fig. 1a). Similarly, in other trypanosomatids the U2 snRNA is encoded by a single-copy gene, with the exception of T. cruzi and L. collosoma that contain three genes [39]. In T. cruzi, only one of the U2 snRNA genes is transcribed [39]. Interestingly, sequence analysis allowed us to determine that the two T. cruzi U2 snRNA genes that are not expressed possess a box $\mathrm{A}$ in the associated tRNA-like sequence, but do not contain a box B (Additional file 5: Figure S5). Thus, the absence of box B in the neighbor tRNA-like sequence might contribute to the silencing of two U2 snRNA genes in T. cruzi. Remarkably, from all the trypanosomatids with genome sequences available, C. fasciculata is the only organism 
that does not have a tRNA-like associated with the U2 snRNA, as it contains a tRNA-Ala gene whose box A is located 105 bp upstream of the U2 snRNA gene (Additional file 6: Figure S6).

\section{Conclusions}

In the present study we have demonstrated that transcription of the $L$. major U2 snRNA gene is dependent on boxes A and B from the associated tRNA-like and a sequence located at the $5^{\prime}$ end of the U2 snRNA. Notably, unlike any other snRNA or 7SL RNA genes in trypanosomatids, box B from the upstream tRNA-Ala is also important for the expression of the L. major U2 snRNA gene. Thus, the U2 snRNA gene promoter shows unique features in L. major. Also, we have mapped several pseudouridines in the L. major U2 snRNA gene, including one located in the Sm binding site that has not been reported in any other organism. Future studies will explore the role that pseudouridines might play in splicing and snRNP assembly in Leishmania.

\section{Additional files}

Additional file 1: Figure S1. Sequence alignment of U2 snRNA genes from trypanosomatids. Sequences were obtained from the TriTrypDB databases of the following species of trypanosomatids: L. major Friedlin (Lmj), L. arabica LEM1108 (Lar), L. tarentolae Parrot-Tarll (Ltr), L. infantum JPCM5 (Lin), L. amazonensis MHOMBR/71973/M2269 (Laz), L. donovani BPK282A1 (Ldn), L. aethiopica L147 (Lae), L. mexicana U1103 (Lmx), L. panamensis MHOM/COL/81/L13 (Lpn), L. braziliensis M2904 (Lbr), L. enriettii LEM3045 (Len), E. monterogeii LV88 (Emg), C. fasciculata Cf-Cl (Cfs), L. pyrrhocoris H10 (Lpy), L. seymouri ATCC 30220 (Lsy), L. collosoma (unspecified strain) [39] (LCl), T. cruzi CL Brener Non-Esmeraldo-like, copy of the chromosome 23 (Tcr), T. vivax Y486 (Tvx), T. congolense IL3000 (Tcn), T. evansi STIB 805 (Tev) and T. brucei DAL972 (Tbr). The alignment was obtained with the DNAMAN software, and manually corrected. Arrows indicate the identified pseudouridines $(\Psi)$ in the L. major U2 snRNA (this work). BPRR and Sm sites are also indicated. The $3^{\prime}$ ends (C residues) that have been experimentally mapped are shown in red font for $L$. major (this work), T. cruzi [45] and T. brucei [44]. The T-tract length is indicated for each species. Sequence numbers are relative to the TSS $(+1)$ from the U2 snRNA. This alignment was used to generate the sequence logo depicted in Fig. 3b. (PDF $2137 \mathrm{~kb})$

Additional file 2: Figure S2. Northern blot analysis of the tagged U2 snRNA. The experiment was performed with total RNA from cells transfected with construct 1 (pComp), construct $6(p B S+6 /+12)$, the unrelated vector pLMRIB or mock-transfected cells. The probe was the oligonucleotide LmjU2tag-Rev, which specifically recognizes the tag sequence. (TIF $807 \mathrm{~kb}$ )

Additional file 3: Figure S3. Mutations introduced in the tRNA-Ala/U2 snRNA locus. For each vector, sequence elements are indicated inside colored boxes: tRNA-Ala in green, tRNA-like in orange and U2 snRNA in blue. Boxes $A$ and $B$ are also indicated. The tag sequence, inserted within the $U 2$ snRNA gene, is also shown. Base substitutions are indicated in red font. (PDF $3122 \mathrm{~kb}$ )

Additional file 4: Figure S4. Predicted secondary structure of the tRNA-Ala (panel a) and the tRNA-like associated to the L. major U2 snRNA gene (panel b). The minimum free energy structures are shown. The color scale indicates low (blue) to high (red) probabilities of base pairing. (PDF $355 \mathrm{~kb}$ )

Additional file 5: Figure S5. Sequence comparisons of U2 snRNA genes and flanking regions from T. cruzi (CL Brener Non-Esmeraldo-like).
Sequences from the genes located on chromosomes 23, 37 and 6 are shown. The U2 snRNA gene from chromosome 23 is presented in blue font. The position of boxes $A$ and $B$ is indicated. Sequence numbers are relative to the TSS (+1) from the U2 SnRNA. (PDF $1404 \mathrm{~kb})$

Additional file 6: Figure S6. Genomic context of U2 snRNA genes in trypanosomatids. Schematic representations of the U2 snRNA loci from Leishmania spp., E. monterogeii (LV88), L. seymouri (ATCC 30220), C. fasciculata (Cf-Cl), T. cruzi (CL Brener Non-Esmeraldo-like, copy of the chromosome 23) and T. brucei (DAL972). All of the U2 snRNA genes contain divergently-oriented boxes $\mathrm{A}$ and $\mathrm{B}$ at conserved distances (around 104 bases), which are contained in tRNA-like sequences. The exception is C. fasciculata, where boxes A and B are located inside a tRNA-Ala gene. In T. cruzi, a tRNA-Arg gene is located downstream of the U2 snRNA gene. Figure is drawn to scale. (TIF $268 \mathrm{~kb}$ )

\section{Abbreviations}

5'-RACE, 5' Rapid Amplification of CDNA Ends; BPRR, branch point recognition region; CMCT, N-cyclohexyl-N'- $\beta$-(4-methylmorpholinium)ethyl-carbodiimide p-tosylate; mRNA, messenger RNA; RNAP, RNA polymerase; RT-qPCR, real-time quantitative polymerase chain reaction; snRNA, small nuclear RNA; tRNA, transfer RNA; TSS, transcription start site

\section{Acknowledgments}

This work is one of the requirements to obtain the PhD degree in Posgrado en Ciencias Biológicas (UNAM) for Saúl Rojas-Sánchez, who was the recipient of doctoral fellowships from CONACyT (Fellowship 256179, CVU 384986), and CEP/UNAM (303180353).

\section{Funding}

This work was supported by grant 251831 from CONACyT, grants IN210712 and IN214715 from PAPIIT (UNAM), and grant FESI-DIP-PAPCA-2014-24 (PAPCA, FES Iztacala) to S. Martínez-Calvillo; and by grants 132312 and 139898 from CONACYT to R. Manning-Cela. The funders had no role in the design of the study, data collection and analysis, or writing of the manuscript.

\section{Availability of data and material}

The datasets supporting the conclusions of this article are included within the article and its additional files.

\section{Authors' contributions}

SRS performed most of the experiments, analyzed data and drafted the manuscript. EFA generated some vectors and analyzed data. RMC performed some in silico analyses and helped perform RT-PCR analysis. LEFM helped perform transfections, Northern blot and RT-qPCR analyses. RGMC participated in the writing and editing of the manuscript. SMC conceived the study, analyzed data and wrote the manuscript. All authors read and approved the manuscript.

\section{Competing interests}

The authors declare that they have no competing interests.

\section{Consent for publication}

Not applicable.

\section{Ethics approval and consent to participate}

Not applicable.

\section{Author details \\ 'Unidad de Biomedicina, Facultad de Estudios Superiores Iztacala, Universidad Nacional Autónoma de México, Av. de los Barrios 1, Col. Los Reyes Iztacala, Tlalnepantla, Edo. de México CP 54090, Mexico. \\ 2Departamento de Biomedicina Molecular, Centro de Investigación y de Estudios Avanzados del IPN, Av. IPN 2508, México, DF CP 07360, Mexico.}

Received: 24 March 2016 Accepted: 5 July 2016

Published online: 19 July 2016

\section{References}

1. Cvitkovic I, Jurica MS. Spliceosome database: a tool for tracking components of the spliceosome. Nucleic Acids Res. 2013;41(Database issue):D132-41. 
2. Chen W, Moore MJ. The spliceosome: disorder and dynamics defined. Curr Opin Struct Biol. 2014;24:141-9.

3. Wahl MC, Will CL, Luhrmann R. The spliceosome: design principles of a dynamic RNP machine. Cell. 2009;136(4):701-18.

4. Leung AK, Nagai K, Li J. Structure of the spliceosomal U4 snRNP core domain and its implication for snRNP biogenesis. Nature. 2011;:473(7348):536-9

5. Zhou L, Hang J, Zhou Y, Wan R, Lu G, Yin P, et al. Crystal structures of the Lsm complex bound to the 3 ' end sequence of U6 small nuclear RNA. Nature. 2014:506(7486):116-20.

6. Rodgers ML, Tretbar US, Dehaven A, Alwan AA, Luo G, Mast HM, et al. Conformational dynamics of stem II of the U2 snRNA. RNA. 2016;22(2):225-36.

7. Ro-Choi TS, Choi YC. Chemical approaches for structure and function of RNA in postgenomic era. J Nucleic Acids. 2012;2012:369058.

8. Adachi H, Yu YT. Insight into the mechanisms and functions of spliceosomal snRNA pseudouridylation. World J Biol Chem. 2014;5(4):398-408.

9. Yu AT, Ge J, Yu YT. Pseudouridines in spliceosomal snRNAs. Protein Cell. 2011;2(9):712-25.

10. Egloff S, O'Reilly D, Murphy S. Expression of human snRNA genes from beginning to end. Biochem Soc Trans. 2008;36(Pt 4):590-4.

11. Mroczek S, Dziembowski A. U6 RNA biogenesis and disease association. Wiley Interdiscip Rev RNA. 2013;4(5):581-92.

12. Orioli A, Pascali C, Pagano A, Teichmann M, Dieci G. RNA polymerase III transcription control elements: themes and variations. Gene. 2012;493(2):185-94.

13. Martinez-Calvillo S, Vizuet-de-Rueda JC, Florencio-Martinez LE, Manning-Cela RG, Figueroa-Angulo EE. Gene expression in trypanosomatid parasites. J Biomed Biotechnol. 2010;2010:525241.

14. Bitar M, Boroni M, Macedo AM, Machado CR, Franco GR. The spliced leader trans-splicing mechanism in different organisms: molecular details and possible biological roles. Front Genet. 2013;4:199.

15. Gunzl A. The pre-mRNA splicing machinery of trypanosomes: complex or simplified? Eukaryot Cell. 2010;9(8):1159-70.

16. Papasaikas P, Valcarcel J. The Spliceosome: The Ultimate RNA Chaperone and Sculptor. Trends Biochem Sci. 2016;41 (1):33-45.

17. Fantoni A, Dare AO, Tschudi C. RNA polymerase III-mediated transcription of the trypanosome U2 small nuclear RNA gene is controlled by both intragenic and extragenic regulatory elements. Mol Cell Biol. 1994;14(3):2021-8.

18. Nakaar V, Dare AO, Hong D, Ullu E, Tschudi C. Upstream tRNA genes are essential for expression of small nuclear and cytoplasmic RNA genes in trypanosomes. Mol Cell Biol. 1994;14(10):6736-42.

19. Djikeng A, Ferreira L, D'Angelo M, Dolezal P, Lamb T, Murta S, et al. Characterization of a candidate Trypanosoma brucei U1 small nuclear RNA gene. Mol Biochem Parasitol. 2001;113(1):109-15.

20. Tschudi C, Ullu E. Unconventional rules of small nuclear RNA transcription and cap modification in trypanosomatids. Gene Expr. 2002;10(1-2):3-16.

21. Nakaar VT, Tschudi C, Ullu E. An unusual liaison: small nuclear and cytoplasmic RNA genes team up with tRNA genes in trypanosomatid protozoa. Parasitol Today. 1995;11:4.

22. Nakaar V, Gunzl A, Ullu E, Tschudi C. Structure of the Trypanosoma brucei U6 snRNA gene promoter. Mol Biochem Parasitol. 1997;88(1-2):13-23.

23. Li L, Otake LR, Xu Y, Michaeli S. The trans-spliceosomal U4 RNA from the monogenetic trypanosomatid Leptomonas collosoma. Cloning and identification of a transcribed tRNA-like element that controls its expression. J Biol Chem. 2000;275(4):2259-64.

24. Robinson KA, Beverley SM. Improvements in transfection efficiency and tests of RNA interference (RNAi) approaches in the protozoan parasite Leishmania. Mol Biochem Parasitol. 2003;128(2):217-28.

25. Martinez-Calvillo S, Sunkin SM, Yan S, Fox M, Stuart K, Myler PJ. Genomic organization and functional characterization of the Leishmania major Friedlin ribosomal RNA gene locus. Mol Biochem Parasitol. 2001;116(2):147-57.

26. Edgar RC. MUSCLE: a multiple sequence alignment method with reduced time and space complexity. BMC Bioinformatics. 2004;5:113.

27. Lorenz R, Bernhart SH, Honer Zu Siederdissen C, Tafer H, Flamm C, Stadler PF, et al. ViennaRNA Package 2.0. Algorithms Mol Biol. 2011;6:26.

28. Carey MF, Peterson CL, Smale ST. The primer extension assay. Cold Spring Harb Protoc. 2013;2013(2):164-73.

29. Martinez-Calvillo S, Nguyen D, Stuart K, Myler PJ. Transcription initiation and termination on Leishmania major chromosome 3. Eukaryot Cell. 2004;3(2):506-17.
30. Motorin Y, Muller S, Behm-Ansmant I, Branlant C. Identification of modified residues in RNAs by reverse transcription-based methods. Methods Enzymol. 2007:425:21-53.

31. Barth S, Hury A, Liang XH, Michaeli S. Elucidating the role of H/ACA-like RNAs in trans-splicing and rRNA processing via RNA interference silencing of the Trypanosoma brucei CBF5 pseudouridine synthase. J Biol Chem. 2005;280(41):34558-68

32. Hofstetter $\mathrm{H}$, Kressman A, Birnstiel ML. A split promoter for a eucaryotic tRNA gene. Cell. 1981;24(2):573-85.

33. Baker RE, Gabrielsen O, Hall BD. Effects of tRNATyr point mutations on the binding of yeast RNA polymerase III transcription factor C. J Biol Chem. 1986;261(12):5275-82.

34. Ivens AC, Peacock CS, Worthey EA, Murphy L, Aggarwal G, Berriman M, et al. The genome of the kinetoplastid parasite, Leishmania major. Science. 2005:309(5733):436-42.

35. Downing T, Imamura H, Decuypere S, Clark TG, Coombs GH, Cotton JA, et al. Whole genome sequencing of multiple Leishmania donovani clinical isolates provides insights into population structure and mechanisms of drug resistance. Genome Res. 2011;21(12):2143-56.

36. Peacock CS, Seeger K, Harris D, Murphy L, Ruiz JC, Quail MA, et al. Comparative genomic analysis of three Leishmania species that cause diverse human disease. Nat Genet. 2007;39(7):839-47.

37. Rogers MB, Hilley JD, Dickens NJ, Wilkes J, Bates PA, Depledge DP, et al. Chromosome and gene copy number variation allow major structural change between species and strains of Leishmania. Genome Res. 2011;21(12):2129-42

38. Raymond F, Boisvert S, Roy G, Ritt JF, Legare D, Isnard A, et al. Genome sequencing of the lizard parasite Leishmania tarentolae reveals loss of genes associated to the intracellular stage of human pathogenic species. Nucleic Acids Res. 2012:40(3):1131-47.

39. Hartshorne T, Agabian N. A new U2 RNA secondary structure provided by phylogenetic analysis of trypanosomatid U2 RNAs. Genes Dev. 1990;4(12A): 2121-31.

40. Tschudi C, Richards FF, Ullu E. The U2 RNA analogue of Trypanosoma brucei gambiense: implications for a splicing mechanism in trypanosomes. Nucleic Acids Res. 1986;14(22):8893-903.

41. Wang P, Palfi Z, Preusser C, Lücke S, Lane WS, Kambach C, et al. Sm core variation in spliceosomal small nuclear ribonucleoproteins from Trypanosoma brucei. EMBO J. 2006;25(19):4513-23.

42. Crooks GE, Hon G, Chandonia JM, Brenner SE. WebLogo: a sequence logo generator. Genome Res. 2004;14(6):1188-90.

43. Padilla-Mejia NE, Florencio-Martinez LE, Figueroa-Angulo EE, Manning-Cela RG, Hernandez-Rivas R, Myler PJ, et al. Gene organization and sequence analyses of transfer RNA genes in trypanosomatid parasites. BMC Genomics. 2009;10:232

44. Hitchcock RA, Zeiner GM, Sturm NR, Campbell DA. The 3' termini of small RNAs in Trypanosoma brucei. FEMS Microbiol Lett. 2004;236(1):73-8.

45. Ambrosio DL, Silva MT, Cicarelli RM. Cloning and molecular characterization of Trypanosoma cruzi U2, U4, U5, and U6 small nuclear RNAs. Mem Inst Oswaldo Cruz. 2007:102(1):97-105.

46. Ben-Shlomo H, Levitan A, Beja O, Michaeli S. The trypanosomatid Leptomonas collosoma 7SL RNA gene. Analysis of elements controlling its expression. Nucleic Acids Res. 1997;25(24):4977-84.

47. Velez-Ramirez DE, Florencio-Martinez LE, Romero-Meza G, Rojas-Sanchez S, Moreno-Campos R, Arroyo R, et al. BRF1, a subunit of RNA polymerase III transcription factor TFIIIB, is essential for cell growth of Trypanosoma brucei. Parasitology. 2015;142(13):1563-73.

48. Broadbent S, van der Woude M, Aziz N. Accurate and simple sizing of primer extension products using a non-radioactive approach facilitates identification of transcription initiation sites. J Microbiol Methods. 2010;81(3):256-8. 